\title{
Quaternion-Based Improved Artificial Bee Colony Algorithm for Color Remote Sensing Image Edge Detection
}

\author{
Dujin Liu, ${ }^{1,2}$ Huajun Wang, ${ }^{1}$ Sen Wang, ${ }^{3}$ Guolin $\mathrm{Pu}^{2}$ Xiaoya Deng, ${ }^{2}$ and Xiang Hou ${ }^{2}$ \\ ${ }^{1}$ College of Geophysics, Chengdu University of Technology, Chengdu 610059, China \\ ${ }^{2}$ School of Computer Science, Sichuan University of Arts and Science, Dazhou 635000, China \\ ${ }^{3}$ State Key Laboratory of Wireless Mobile Communications, China Academy of Telecommunications Technology, Beijing 100191, China
}

Correspondence should be addressed to Huajun Wang; wanghuajun02@126.com

Received 12 June 2014; Revised 13 August 2014; Accepted 24 August 2014

Academic Editor: Wai Yuen Szeto

Copyright (C) 2015 Dujin Liu et al. This is an open access article distributed under the Creative Commons Attribution License, which permits unrestricted use, distribution, and reproduction in any medium, provided the original work is properly cited.

\begin{abstract}
As the color remote sensing image has the most notable features such as huge amount of data, rich image details, and the containing of too much noise, the edge detection becomes a grave challenge in processing of remote sensing image data. To explore a possible solution to the urgent problem, in this paper, we first introduced the quaternion into the representation of color image. In this way, a color can be represented and analyzed as a single entity. Then a novel artificial bee colony method named improved artificial bee colony which can improve the performance of conventional artificial bee colony was proposed. In this method, in order to balance the exploration and the exploitation, two new search equations were presented to generate candidate solutions in the employed bee phase and the onlookers phase, respectively. Additionally, some more reasonable artificial bee colony parameters were proposed to improve the performance of the artificial bee colony. Then we applied the proposed method to the quaternion vectors to perform the edge detection of color remote sensing image. Experimental results show that our method can get a better edge detection effect than other methods.
\end{abstract}

\section{Introduction}

Edge detection in remote sensing image is very important for a wide range of applications such as environment monitoring [1], image analysis and classification [2,3], change detection [4], and geographic image retrieval [5].

With the development of remote sensing technology, a large number of remote sensing images with high spectral and spatial resolutions are acquired by the satellites, and the revisiting time is greatly reduced. As a result, the users obtain the larger archives of digital remote sensing image, which facilitates the edge detection of remote sensing image. However, there is still a challenging problem to achieve reliable image edges in remote sensing image, not only due to the large amount of data and the rich image details, but also because of the containing of too much noise.

Artificial bee colony algorithm (ABC) [6] is widely used in image processing $[7,8]$ and engineering optimization
$[9,10]$. For example, Draan and Bouaziz [11] proposed a new artificial bee colony $(\mathrm{ABC})$ algorithm for image contrast enhancement. The proposed algorithm has been extended to color image enhancement and given very promising results. Szeto and Jiang [12] proposed a hybrid enhanced artificial bee colony algorithm (HEABC) for solving the problem of bus network design. The HEABC relies mainly on the enhanced artificial bee colony algorithm to determine the route structure and could produce a design that was better than the existing design for maximum intermediate stops, total travel time, number of transfers, maximum headway, and total fuel cost. But it has not yet been improved to solve remote sensing image edge detection problem. This study enhances the $\mathrm{ABC}$ algorithm to solve this problem.

The color image can provide a large amount of appearance information of the object. However, conventional ABC cannot take full advantage of the color image. Now, there are many kinds of color remote sensing image edge detection 
methods $[13,14]$, but each method performed remote sensing image edge detection by splitting the strong spectral relationship between each channel of the color image [15]. The vector method [16] takes a color image pixel as a three-dimensional vector, which to some extent considered each channel nature of the color image making the effect better than the synthetic method [17]. But the vector method does not process a color as a whole in transformation, operation, and treatment of the image vector, which lead to some edges undetected, local color uncoordinated, and local edge details damaged. It is even worse for the effect when the complex noise exists [18].

How to choose a mathematic tool to effectively represent a color image, which makes the three color components be considered simultaneously as a whole without loss of the spectral relation that is present in them, is a challenging problem.

It is a good way to use a quaternion [19] representing a color pixel. A quaternion can be seen as a composition of four real numbers. We can let the first one of the four real numbers be zero for representing a pixel, and let the other three real numbers be, respectively, the values of the three entries of a color pixel. Through quaternion representation of color image, you can make full use of the whole color information for each pixel of the image. A color image can be represented as a quaternion matrix, while each pixel of the color image is represented by a quaternion vector. Then according to quaternion vector rotation principle and tone offset mechanisms, the edge points can be determined [20], which implements the whole processing of each quaternion pixel. In recent years, there have been a number of studies on the quaternion $[21,22]$.

In this paper, we aim at using the quaternion to represent the color pixel and reducing the dimension for multispectral remote sensing images by using PCA (principal component analysis) $[23,24]$. Then we propose a novel ABC method called IABC (improved artificial bee colony) and use it for the image edge detection, based on which, aquaternion-based improved artificial bee colony algorithm for edge detection of color remote sensing image will be introduced.

The remainder of the paper is organized as follows. In Section 2, quaternion representation for a color image edge will be present. In Section 3, we introduce our algorithm. In Section 4, we do some experiments and analyze their results. In Section 5, conclusion is offered.

\section{Quaternion Representation for the Color Image Edge}

Although the quaternion theory [19] still has some issues to be resolved, its representation is convenient, simple, and widely used in image processing. Based on quaternion theory, a quaternion is a four-dimensional number, which consists of a real part and three imaginary parts. For example, the quaternion $q$ can be expressed as follows:

$$
q=a+b i+c j+d k
$$

where $a, b, c$, and $d$ are four real coefficients. And $i, j$, and $k$ are three orthogonal imaginary units that satisfy the following rules:

$$
\begin{gathered}
i^{2}=j^{2}=k^{2}=i j k=-1, \\
i j=k, \quad j k=i, \quad k i=j, \\
j i=-k, \quad k j=-i, \quad i k=-j .
\end{gathered}
$$

Let the real part of quaternion $a=0$; then $q$ is a pure quaternion. Let $\operatorname{Re}(q)=a$ represent the real part of quaternion $q$, and let $\operatorname{Im}(q)=b i+c j+d k$ represent imaginary part of quaternion $q$. Thus $q=\operatorname{Re}(q)+\operatorname{Im}(q)$. Mold and conjugate of quaternion $q$ are defined as follows:

$$
\begin{gathered}
|q|=\sqrt{a^{2}+b^{2}+c^{2}+d^{2}}, \\
\bar{q}=a-b i-c j-d k .
\end{gathered}
$$

Denote each color pixel of a color image by quaternion $q=a+b i+c j+d$ as follows: $a, b, c$, and $d$ are set as $a=0$, $b=r, c=g$, and $d=b . r, g$, and $b$ stand for the first, second, and third components of the color pixel, respectively.

Addition or subtraction with two quaternions is addition or subtraction of their respective components, and according to the rules of quaternion multiplication, the quaternion does not satisfy the multiplication commutative law.

2.1. Polar Coordinates for Quaternions. Feature axis and feature angle of quaternion $q$ are defined as follows:

$$
\begin{gathered}
\mu=\frac{1}{\sqrt{b^{2}+c^{2}+d^{2}}}(b i+c j+d k), \\
\theta= \begin{cases}\tan ^{-1}\left(\frac{\sqrt{b^{2}+c^{2}+d^{2}}}{a}\right), & a \neq 0 \\
\frac{\pi}{2}, & a=0,\end{cases}
\end{gathered}
$$

where $q=a+b i+c j+d k$

Where $q$ can be derived after calculation as follows: $q=$ $|q|(\cos \theta+\mu \sin \theta)=|q| e^{\mu \theta}$, meanwhile $u$ denotes the eigenaxis, and $\theta$ denotes the eigenangle.

If vector $\alpha$ rotating angle $\theta$ becomes vector $\beta$ and $e_{n}$ denotes unit vector of rotating shaft direction perpendicular to the plane which includes vectors $\alpha, \beta$, rotating shaft direction meets the right-hand rule, and then a quaternion will be represented in polar coordinates as follows:

$$
\beta=\left(\cos \theta+e_{n} \sin \theta\right) \alpha
$$

And the corresponding formula of the reverse rotation is as follows:

$$
\beta=\left(\cos (-\theta)+e_{n} \sin (-\theta)\right) \alpha .
$$


If the vector $\alpha$ is not perpendicular to the rotary shaft $e_{n}$ and it rotates angle $\theta$ about the axis of rotation becoming the vector $\beta$, the quaternion in polar coordinates is expressed as

$$
\beta=\left(\cos \frac{\theta}{2}+e_{n} \sin \frac{\theta}{2}\right) \alpha\left(\cos \frac{\theta}{2}-e_{n} \sin \frac{\theta}{2}\right),
$$

where every color pixel denoted by a quaternion is a threecomponent vector of integer values in the interval $[0,255]$.

Let $x_{i j}=\left[r_{i j}, g_{i j}, b_{i j}\right]^{T}$, where $r_{i j}, g_{i j}, b_{i j} \in\{0,1,2, \ldots, 255\}$. For reasons of symmetry, we transfer the RGB color space from the coordinate origin $(0,0,0)$ to the center $(127.5,127.5,127.5)$ of the RGB space. Then pure imaginary quaternion of a color pixel $x_{i j}$ is expressed as $q_{i j}=\left(r_{i j}-\right.$ $127.5) i+\left(g_{i j}-127.5\right) j+\left(b_{i j}-127.5\right) k$, and any unit quaternion $U$ can be represented as $R=e^{u \theta}=\cos \theta+\mu \sin \theta$. According to the three-dimensional rotation quaternion principle mentioned above, $R X R^{*}$ indicates a three-dimensional vector $X$ (represented by purely imaginary quaternions) rotating an angle $2 \theta$ around the $U$-axis $[25,26]$.

2.2. Quaternion Representation of Color Image Edge Points. If color pixels in RGB three-dimensional space are expressed as vectors, the vector modulus value of every pixel would indicate brightness of this pixel, of which direction means the color hue and saturation of the pixel. In order to detect image edge, grayscale pixel vector $\mu=i+j+k$ would be regarded as the rotation axis; that is, when $\theta=\pi / 2$, it can be expressed in polar coordinates as $R=e^{u \theta}=i+j+k$. So for any pure imaginary quaternion vector $X, R X R^{*}$ indicates three-dimensional vector $X$ rotating $2 \theta$ angle $\left(180^{\circ}\right)$ around the axis $\mu$, which means $X$ is relative to the vector $\mu$ on the axis of symmetry in the opposite direction. According to this principle, for any one of the color pixel vector $q$, there is $q+$ $R q R^{*}$ being a purely imaginary quaternion and $\left|q+R q R^{*}\right|=$ 0 . Then for any two color pixel vectors $q_{1}=r_{1} i+g_{1} j+b_{1} k$ and $q_{2}=r_{2} i+g_{2} j+b_{2} k$, we assume $q_{3}=q_{1}+R q_{2} R^{*}$. If the two vectors $q_{1}, q_{2}$ are of the same color pixel, $q_{3}$ will be a gray pixel vector, which means the direction coincides with the direction of the rotation axis $u$. If the two vectors $q_{1}, q_{2}$ are not identical, the resultant vector $q_{3}$ will deviate from the rotation axis $u$ and be a color pixel. If the hues of $q_{1}$ and $q_{2}$ are close, $q_{3}$ will be located near the gray vector $\mu$. In other words, if there is an arbitrarily small positive number $\delta$, there is $\left|q_{1}+R q_{2} R^{*}\right|<\delta(\delta$ is an arbitrary small positive number). By this method, if these two color pixel vectors $q_{1}$ and $q_{2}$ correspond with the pixels in the image edge, it can be naturally judged [27].

In fact, for grayscale images, the edges can be considered as brightness discontinuous jump, and for color images, the edge not only has the mutation of the brightness (even the same color on both sides of the border), but also should meet the condition that different color regions are on both sides of the edges (even if the same brightness on both sides of the border). Thus the edge detection problem is to find out the pixel points meeting these two conditions, that is, to detect a pixel set not in the local area of the same color but connecting the pixels of local same color area. If there are two pixels, when a pixel is on the edge, the other pixel is outside the edge, we rotate one pixel $180^{\circ}$ around the gray axis $u=i+j+k$ and add it to the other one. The result represents a pixel which must be color. On this basis, for any pixel $q=r i+g j+b k$, in order to determine whether the pixel is on the edge, we can use the left template and the right template [28] to perform convolution operation as follows:

$$
\left[\begin{array}{lll}
\beta & \beta & \beta \\
0 & 0 & 0 \\
1 & 1 & 1
\end{array}\right]\left[\begin{array}{ccc}
c_{1} & c_{2} & c_{3} \\
0 & 0 & 0 \\
c_{4} & c_{5} & c_{6}
\end{array}\right]\left[\begin{array}{ccc}
\beta^{\prime} & \beta^{\prime} & \beta^{\prime} \\
0 & 0 & 0 \\
1 & 1 & 1
\end{array}\right],
$$

where $c_{1}, c_{2}, c_{3}, c_{4}, c_{5}$, and $c_{6}$ are pixels in the image, while

$$
\begin{gathered}
\beta=\cos \frac{\theta}{2}+u \sin \frac{\theta}{2}, \quad \beta^{\prime}=\cos \frac{\theta}{2}-u \sin \frac{\theta}{2}, \\
u=i+j+k .
\end{gathered}
$$

Equation (8) means that the pixels $c_{1}, c_{2}$, and $c_{3}$ around the gray axis $u$ rotate $180^{\circ}$ according to the right hand rule, and then $c_{4}, c_{5}$, and $c_{6}$ will be, respectively, added. Finally, the result obtained is as follows:

$$
c^{\prime}(m, n)=\left(\beta c_{1} \beta^{\prime}+c_{4}\right)+\left(\beta c_{2} \beta^{\prime}+c_{5}\right)+\left(\beta c_{3} \beta^{\prime}+c_{6}\right) .
$$

If the three color components of the vector $c^{\prime}(m, n)$ are equal or approximately equal, then it will be a local pixel of the same color, and if not, it will be an edge pixel. According to this method, we can determine whether a color pixel is an edge point. Our algorithm will be established on the basis of this method.

\section{Quaternion-Based Improved Artificial Bee Colony Algorithm for Color Remote Sensing Image Edge Detection}

In order to solve the optimization problems characterized by the nonconvex, the discontinuous, as well as the nondifferentiable, and so on, much effort has been made during the past decades, which lead to some great success in evolutionary algorithms. The most prominent evolutionary algorithms are particle swarm optimization (PSO) [29], ant colony optimization (ACO) [30], genetic algorithm (GA) [31], biogeography-based optimization (BBO) [32], and so on.

$\mathrm{ABC}$ has better performance comparing with populationbased algorithms such as GA, DE, and PSO. Now, there have been several types of improvements for conventional $\mathrm{ABC}$ algorithms. Here we mention a few. The first type of improvement is Rosenbrock $\mathrm{ABC}$ (RABC) [33], whose main idea is giving two alternative phases of RABC: the exploration phase realized by $\mathrm{ABC}$ and the exploitation phase completed by the rotational direction method. The second one is taking advantage of the information of the best solutions [34, 35]. The third one [36] proposed an interactive $A B C$, which introduced the concept of universal gravitation into the consideration of the affection between the employed bees and the onlooker bees. But cannot obtain the desired balance between the exploration and the exploitation, convergence of all $\mathrm{ABC}$ algorithms is relatively poor when solving complex problems. 
3.1. Artificial Bee Colony Algorithm. In ABC, bee behavior has the features of autonomy, division of labor, self-organization, and other features. Colony system includes the following elements: bees, honey, and information exchange mechanisms between individuals. Quality of nectar is mainly decided by several aspects: the abundance of nectar, the distance between the point of nectar and bee, and the angle formed between the nectar and sunlight. Worker bees in colonies can be divided into employed bees, onlookers, and scouts. Half of the colony consists of employed bees, and the other half includes onlooker bees. Gathering honey is the basis of survival of swarm, and the basic behaviors of bees are searching nectar, guiding employed bees to the qualified nectar and abandoning the inferior one. The quality and quantity of nectar represent the honey benefits. Finding the most effective source of nectar is a process of exploring the optimal solution. Employed bees master the information of nectar source with high efficiency indexes. Then they pass their food information to the onlooker bees and guide them. But sometimes they exploit the bee nectar themselves. Onlookers are obliged to select good food sources from those found by employed bees to further search the foods. If the food source is abandoned by its employed bees, the employed bees will become the scouts and start to search for a new food source in the vicinity of the hive. Exchange of information between individuals is achieved by the individual dance in the hive dance area.

In $\mathrm{ABC}$, location of a food source corresponds to a possible optimal solution. The quantity of the sources of honey represents their fitness of a possible solution. The number of employed bees equals to the number of food sources. At the initialization step, ABC generates a randomly distributed initial population $L$ of EB solutions (food source positions), where EB denotes the size of employed bees or onlooker bees. The initial solution as follows:

$C X_{i}=\left\{c x_{i, 1}, c x_{i, 2}, \ldots c x_{i, D}\right\}$ is produced randomly in the range of the borders with the parameters as follows:

$$
C X_{i, j}=C X_{l, j}+\beta\left(C X_{u, j}-C X_{l, j}\right), \quad \beta \in[-1,1], u \neq l,
$$

where $i=1,2, \ldots, \mathrm{EB}, j=1,2, \ldots, D$, and $D$ is the problem dimension; $C X_{u, j}$ and $C X_{l, j}$ are the lower and upper borders for the dimension $j$, respectively.

After the initialization step, the population is constantly changing. The employed bees, onlooker bees, and scout bees constantly repeat their search process, which means each employed bee constantly explores new locations and remembers the old best position. If an employed bee finds a new food source that has equal or better quality than the old food source, the old food source will be replaced by the new food source. Otherwise, the old food source will be retained. Each onlooker chooses a food source depending on the probability $p_{i}$ associated with its fitness fit ${ }_{i}$ :

$$
p_{i}=\frac{\mathrm{fit}_{i}}{\sum_{t=1}^{\mathrm{EB}} \mathrm{fit}_{t}},
$$

where fit ${ }_{i}$ is fitness of solution $i$.
ABC finds a new food location from the old best food location by the following equation:

$$
C V_{i, j}=C X_{i, j}+\mu_{i, j}\left(C X_{i, j}-C X_{k, j}\right),
$$

where $k \neq i k \in\{1,2, \ldots, \mathrm{EB}\} j \in\{1,2, \ldots, D\} . k$ and $j$ are randomly chosen indexes, $\mu_{i, j}$ is a random number, and $\mu_{i, j} \in$ $[-1,1]$.

If a position cannot be improved further over a predetermined number of cycles, then that food source will be abandoned, and the scout will try to discover a new food source randomly as in (13) to replace $C X_{i}$.

3.2. Two New Improved Search Equations. How to improve the performance of the $\mathrm{ABC}$ algorithm for balancing between two contradictory aspects of their performance: exploration and exploitation? The key is to improve the search equation. So far there has appeared several improved search equation $[35,37]$. But they only improve the performance in certain specific areas. The most representative algorithm is Gbestguided artificial bee colony algorithm GABC [34]. It proposed the search equation as follows:

$$
C V_{i, j}=C X_{i, j}+\sigma_{i, j}\left(C X_{\text {best }, j}-C X_{i, j}\right)+\mu_{i, j}\left(C X_{i, j}-C X_{k, j}\right) .
$$

The method add the second term in the right side of (14), $C X_{\text {best }, j}$ is the $j$ th element of the global best solution, and $\sigma_{i, j}$ is a random number within $[0,1.5]$. But the experiment results show that this algorithm improvement of the performance is not obvious.

Convergence performance of all the current search equations is not ideal. In this paper, inspired by PSO and combining (13) with (14), we proposed a new improved ABC algorithm (IABC), where two novel search equations are proposed for employed bees and onlookers, respectively, as follows:

$$
\begin{array}{r}
C V_{i, j} \\
=C X_{i, j}+\sigma_{i, j}\left(C X_{\text {best }, j}-C X_{i, j}\right)+\mu_{i, j}\left(C X_{m, j}-C X_{k, j}\right), \\
C V_{i, j}=C X_{m, j}+\tau_{i, j}\left(C X_{\text {best }, j}+\omega\right)+\mu_{i, j}\left(C X_{i, j}-C X_{k, j}\right), \\
p=0.2,
\end{array}
$$

where $k, m, i$, and best are not equal to each other, $k, m \in$ $\{1,2, \ldots \mathrm{EB}\}$, and $j \in\{1,2, \ldots D\} . \sigma_{i, j}$ is a random number in the range of $[0,1.5] \cdot \tau_{i, j}$ is a random number in the range of $[0,1] . \mu_{i, j}$ is a random number, $\mu_{i, j} \in[-1,1]$, and $\omega=$ $p^{2} /\left(C X_{\text {best }, j^{2}}+p^{2}\right)$.

The main difference is that the GABC algorithm employs one search equation (14), but the IABC algorithm employs two different search equations (15) and (16) based on different emphases.

Note that (15) has some similarity with (14). Only the third term is different in (14) and (15); in other words, only the 
subscripts of the third term $i$ and $m$ are different in (14) and (15). In (15), $X_{m}$ is a randomly selected individual from the population, which is more diversity and easier to converge. It can bring a more promising candidate solution compared with (15) and (14). Equation (16) is proposed for onlookers. The first term and the second term have changed in (16) compared with (14), while the subscripts of the first term $i$ and $m$ are different in (14) and (16). $C X_{m, j}$ instead of $C X_{i, j}$ can improve the exploration capability. Experimental results show that the larger value for coefficient $\sigma_{i, j}$ leads to the worse solution quality in a certain range, that is why $\tau_{i, j}$ instead of $\sigma_{i, j}$ can improve the quality of the solution. In (16), $\omega$ instead of $C X_{i, j}$ can not only enhance the exploitation but also improve search efficiency. Thus, (16) may be beneficial to not only the convergence, but also the quality of the solution. That is to say, (15) focuses on the exploration, while (16) emphasizes on the exploitation. The emphases of (15) are consistent with the employed bees stage, and the ones of (16) are consistent with the onlookers stage.

3.3. We Proposed the Algorithm. Based on the improved colony algorithm, firstly we set the conditions for the end of the cycle (according to actual situation, we probably set a smaller number of cycles, different times, or the conditions for the end of the first $\mathrm{N}$-effectiveness values, which are greater than a present threshold). According to the conditions, if IABC does not converge and gets some local optimal solutions, these local optima will correspond to the point on the edge of the image. Continue searching until we get the global optimal solution. The global optimal solution and all the local optima constitute edge points of this remote sensing image. Mark all these points, and then we can form the edge of the image.

Algorithm process is as follows.

(1) At initialization colony step, we randomly generate $n$ bees including employed bees and onlookers and all the employed bees randomly search the nectars. At last we set the stopping condition (subjective effects or maximum number of iterations).

(2) According to the fitness function, we evaluate the (corresponding nectar sources) fitness indicators of individual bees.

(3) When the stopping condition is not satisfied, we select the first $S_{n}$ bees with the highest value of fitness to be the employers. Then the employed bees hired $S_{o}$ onlookers, which will follow the employed bees to the neighborhood of $S_{n}$ nectar sources to search for new nectar source locations, and assess the efficiency indicators.

(4) If a position cannot be improved further over a predetermined number of cycles, then that food source will be abandoned, and the current position will be retained; otherwise, the employer bees will become scout ones.

(5) Scouts randomly search the nectars and evaluate their value of fitness.
(6) When setting condition, we update all the local nectar pbest $(i)(i=1,2,3, \ldots, n)$ and the global nectar gbest (i) $(i=1,2,3, \ldots, N)$.

(7) Set $i=i+1$ and return to (2) until $i=$ MAX or reach the stop condition.

(8) Mark position corresponding to the global optimal solution and all the local optima.

(9) End.

3.4. The Fitness Function. In the image, the function which is strongly associated with the information of edge points can be used for IABC algorithm fitness. Pixel values correspond to the higher fitness, the greater possibility that the pixel can be the edge point. Fitness function can refer to pheromone function of the literature [38].

3.5. Parameter Settings. In the conventional ABC, the colony of artificial bees contains three types of bees: employed bees, onlookers, and scouts. Half of the colony consists of employed bees, and the other half includes onlooker bees. But in fact, we use $\mathrm{ABC}$ to perform image edge detection, and changing the parameters has a greater impact on the image edge detection effect. Above all parameter setting is crucial. Based on the experimental results, we adjust the parameters of the conventional $A B C$ as follows.

Let the number of all the worker bees be $S$ with a more reasonable value range $600 \leq S \leq 1600$. Let the number of employed bees be $S_{e}$, where $0.35 S \leq S_{e} \leq 0.55 S$. If $S$ and $S_{e}$ are too small, it will result in the detection of the image that has missed too many points. If $S$ is too large for image edge detection without obvious contribution, that will only waste more time. Set onlookers scale $S_{o}$, where $0.40 S \leq S_{o} \leq 0.60 S$; let the number of scouts be $S_{s}$, where $0.05 S \leq S_{s} \leq 0.10 S$. If $S_{s}$ is too small, it will increase the algorithm difficulties for jumping out of local optimal solution. If too large, it will increase search computation. In each iteration, bees can be moved along 8-direction of the neighborhood. After calculating the maximum probability for bees of 8-direction of the neighborhood, we select the next locations and steps. Fitness values of bees on each pixel constitute a matrix of fitness values. After the bees had flied to the appropriate number of steps, the fitness value matrix is updated.

\section{Experiments and Results}

To verify the efficiency of the proposed method in color remote sensing image edge detection, experiments have been carried on with a lot of color remote sensing images, which are obtained in actual experiments. In this section, there are two experiments. In the first experiment, we assess the impact caused by the various numbers of different bees on the image edge detection. In the second experiment, we compare our method to other methods on color remote sensing image edge detection. However the results always show that our method is better. 


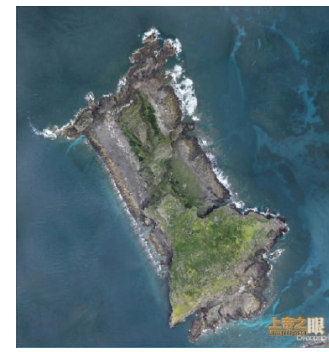

(a)

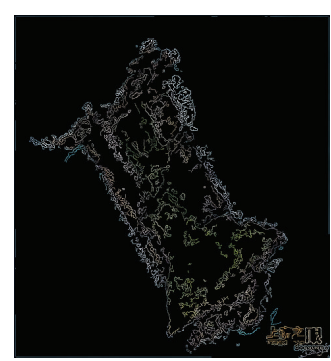

(b)

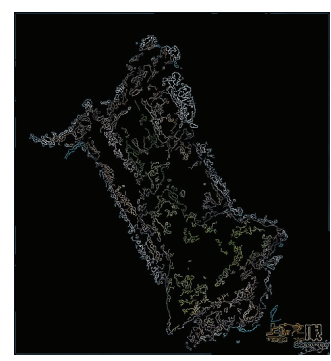

(c)

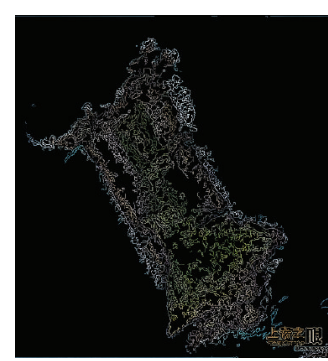

(d)

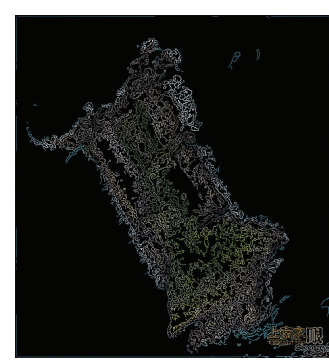

(e)

Figure 1: Performance evaluation: (a) "island” original image, (b) "island" image edge detection result with 600 bees, (c) "island" image edge detection result with 800 bees, (d) "island" image edge detection result with 1200 bees, and (e) "island" image edge detection result with 1600 bees.

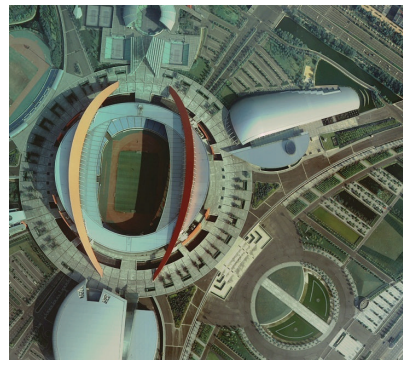

(a)

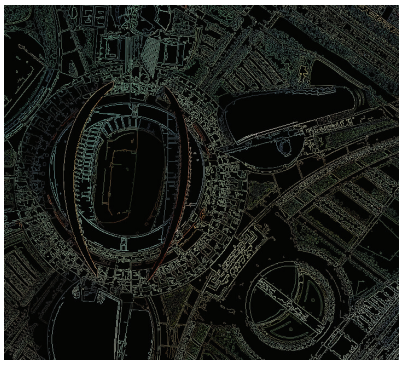

(b)

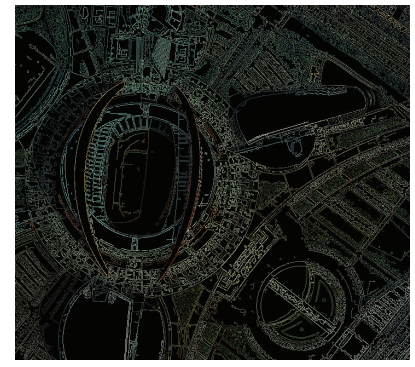

(c)

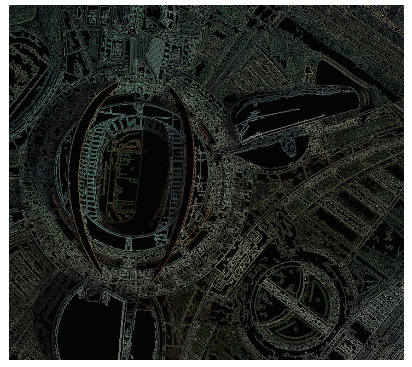

(d)

FIGURE 2: "Sports center" original image and the three algorithms results: (a) "sports center" original image, (b) ABC of nonquaternion representation result image, (c) $\mathrm{ABC}$ of quaternion representation result image, and (d) IABC of quaternion representation result image.

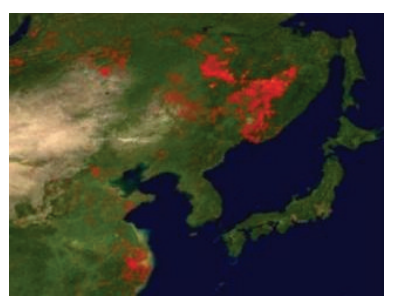

(a)

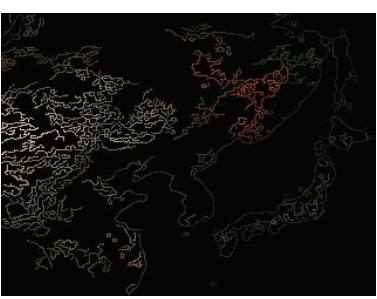

(b)

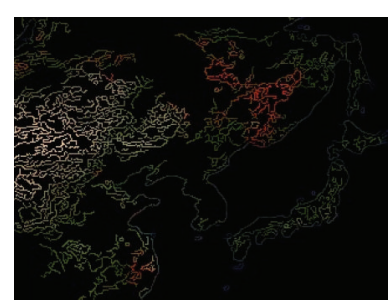

(c)

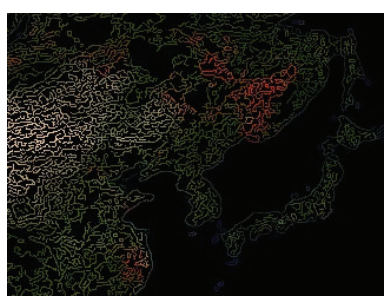

(d)

FIGURE 3: "Forest fires" original image and the three algorithms results: (a) "forest fires" original image, (b) ABC of nonquaternion representation result image, (c) $\mathrm{ABC}$ of quaternion representation result image, and (d) IABC of quaternion representation result image.

4.1. Assess the Impact Caused by the Various Numbers of Different Bees on Color Remote Sensing Image Edge Detection. In this experiment, we use the remote sensing image of an island. Select a $480 \times 521$ pixel area as the study area. Set the number of employed bees $S_{e}=0.50 S$, the number of onlookers $S_{o}=0.45 S$, and the number of scouts $S_{s}=0.05 S$; when the bees' scale $S$ was $600,800,1200$, and 1600 , we perform the image edge detection. In Figures 1(b)-1(e), it, respectively, shows the image edge detection result with $s=$ $600, s=800, s=1200$, and $s=1600$. In Figure 1, when the colony size $S$ is relatively small such as $s=600, s=800$, the edge of the island is affected by noise and image texture as shown in Figures 1(b)-1(c). There is a clear phenomenon of false edges and missed points leading to rough edges. Additionally, color contrast of shallow water area at the edge of the island is not obvious making it unable to be extracted. However when the number of bees is set 1200 and 1600, not only the island edges can be more clearly extracted and much less noise exists, but also the shallow edge of the island can be extracted. As seen from Figure 1, when the remote sensing image detail in the image is not too complicated or rich, the colony number can be reduced appropriately.

\subsection{Experiment on IABC Algorithm for Remote Sensing Images} Edge Detection. In this experiment, we use different types of color remote sensing images to illustrate the effect of our method, including the image of sports center and the image of forest fires. We, respectively, select $728 \times 656$ and $325 \times 245$ pixel region as research subjects, where $S=1600$. This study is 
TABLE 1: Comparison of three algorithms on time in sports center image.

\begin{tabular}{|c|c|c|c|}
\hline \multirow{2}{*}{ Times } & \multicolumn{2}{|c|}{ Quaternion representation } & \multirow{2}{*}{$\begin{array}{c}\text { Nonquaternion } \\
\text { representation } \\
\text { ABC } \\
\end{array}$} \\
\hline & $\mathrm{IABC}$ & $\mathrm{ABC}$ & \\
\hline 1 & 122.21 & 160.71 & 294.03 \\
\hline 2 & 124.03 & 155.94 & 293.12 \\
\hline 3 & 118.20 & 160.37 & 283.59 \\
\hline 4 & 121.11 & 156.56 & 298.45 \\
\hline 5 & 122.37 & 167.01 & 303.23 \\
\hline 6 & 122.79 & 160.32 & 301.09 \\
\hline 7 & 118.19 & 154.52 & 287.43 \\
\hline 8 & 120.49 & 159.01 & 292.16 \\
\hline 9 & 118.66 & 161.75 & 294.62 \\
\hline 10 & 118.23 & 155.36 & 308.10 \\
\hline 11 & 121.21 & 158.19 & 303.22 \\
\hline 12 & 122.49 & 154.32 & 295.98 \\
\hline 13 & 122.20 & 157.50 & 289.56 \\
\hline 14 & 119.18 & 152.29 & 303.09 \\
\hline 15 & 119.49 & 158.98 & 349.92 \\
\hline 16 & 121.29 & 159.22 & 269.85 \\
\hline 17 & 118.20 & 153.09 & 289.72 \\
\hline 18 & 119.17 & 153.45 & 305.89 \\
\hline 19 & 117.78 & 155.99 & 319.04 \\
\hline 20 & 119.32 & 154.93 & 343.24 \\
\hline 21 & 120.35 & 158.92 & 321.03 \\
\hline 22 & 119.24 & 160.24 & 313.32 \\
\hline 23 & 118.90 & 152.92 & 321.49 \\
\hline 24 & 119.92 & 158.39 & 294.34 \\
\hline 25 & 118.17 & 163.96 & 302.44 \\
\hline 26 & 117.79 & 152.24 & 292.37 \\
\hline 27 & 119.95 & 155.76 & 300.20 \\
\hline 28 & 125.39 & 154.04 & 299.13 \\
\hline 29 & 116.89 & 157.84 & 321.50 \\
\hline 30 & 120.25 & 154.42 & 345.67 \\
\hline 31 & 119.64 & 156.23 & 323.89 \\
\hline 32 & 117.93 & 158.60 & 317.21 \\
\hline 33 & 118.42 & 153.33 & 340.26 \\
\hline 34 & 122.72 & 157.50 & 321.29 \\
\hline 35 & 120.29 & 161.34 & 334.03 \\
\hline 36 & 121.83 & 152.99 & 304.18 \\
\hline 37 & 120.30 & 161.76 & 298.01 \\
\hline 38 & 121.49 & 158.54 & 313.94 \\
\hline 39 & 118.07 & 155.62 & 298.99 \\
\hline 40 & 120.80 & 154.27 & 302.95 \\
\hline 41 & 118.56 & 162.04 & 299.48 \\
\hline 42 & 119.98 & 163.76 & 287.65 \\
\hline 43 & 117.95 & 164.03 & 269.24 \\
\hline 44 & 119.22 & 163.62 & 294.67 \\
\hline
\end{tabular}

TABle 1: Continued.

\begin{tabular}{llcc}
\hline Times & \multicolumn{2}{c}{ Quaternion representation } & $\begin{array}{c}\text { Nonquaternion } \\
\text { representation }\end{array}$ \\
& IABC & ABC & ABC \\
\hline 45 & 117.92 & 159.32 & 325.24 \\
46 & 116.75 & 162.92 & 331.08 \\
47 & 118.91 & 153.02 & 332.12 \\
48 & 122.34 & 164.36 & 302.79 \\
49 & 121.80 & 161.29 & 289.70 \\
50 & 120.24 & 154.40 & 291.46 \\
\hline Mean & 119.97 & 157.94 & 306.3 \\
\hline
\end{tabular}

divided into two parts. The first part is a subjective evaluation, and three algorithms were carried out on two remote sensing images. Edge detection results are shown in Figures 2(b)2(d) and Figures 3(b)-3(d). From left to right, there are sequentially $A B C$ of nonquaternion representation result image, $\mathrm{ABC}$ of quaternion representation result image, and $\mathrm{IABC}$ of quaternion representation result image in Figures 23.

As seen in Figures 2(b)-2(d), Figure 2(d) is the best, while Figure 2(b) is the worst. In Figures 2(b)-2(c), there have been a considerable number of undetected points and wrong points. But in Figure 2(d), there are less wrong points, and the entire structure, contour of sports center image, and the runway have been clearly detected. In Figures 2(c)-2(d) and Figures 3(c)-3(d), noise is effectively suppressed. This is mainly due to using quaternions to describe the vectors of color pixels, making noise point be a singular point. So it is easy to separate the noise points and general pixels. In Figure 3(b), the effect of image edge detection is the worst. The red part of the forest fire edge line is interrupted, and the river edge in the forest fire image is not even completely extracted. In Figures 3(c)-3(d), the effect of image edge detection is relatively better. Additionally image edge detection effect in Figure 3(d) is even better and the forest fire edges line is more complete, the edge of the forest and the river's edge is more clear. Even the noise is also smaller.

The second part of the experiment is the one about the objective. By summarizing and analysing the numerical results listed in Tables 1-5, some conclusions can be drawn.

After analysing the time and quantity of the two remote sensing images and independently performing each of these three algorithms 50 times, in terms of time, the results are shown in Tables 1 and 2 . In terms of quantity, according to the literature [39], we use quantitative analysis method to perform the image edge detection. This is a typical method to evaluate the effect of image edge detection. The experimental data is shown in Tables 3-5.

As seen from Tables 1 and 2, for color images using quaternion representation, compared with the average time, in Table 1, average running time of IABC is $75.96 \%$ of $A B C$ and is $39.21 \%$ of $A B C$ based on nonquaternion representation. In Table 2, for color images using quaternion representation, average running time of IABC is $75.01 \%$ of $A B C$ and is $41.45 \%$ of $\mathrm{ABC}$ based on the nonquaternion representation. For color 
TABLE 2: Comparison of three algorithms on time in forest fires image.

\begin{tabular}{|c|c|c|c|}
\hline \multirow{2}{*}{ Times } & \multicolumn{2}{|c|}{ Quaternion representation } & \multirow{2}{*}{$\begin{array}{c}\text { Nonquaternion } \\
\text { representation } \\
\text { ABC } \\
\end{array}$} \\
\hline & $\mathrm{IABC}$ & $\mathrm{ABC}$ & \\
\hline 1 & 26.12 & 38.33 & 63.22 \\
\hline 2 & 24.16 & 35.25 & 62.35 \\
\hline 3 & 25.38 & 36.29 & 64.92 \\
\hline 4 & 24.67 & 35.47 & 63.27 \\
\hline 5 & 27.24 & 34.90 & 66.78 \\
\hline 6 & 27.38 & 37.23 & 62.20 \\
\hline 7 & 24.35 & 38.89 & 70.39 \\
\hline 8 & 29.27 & 35.47 & 65.32 \\
\hline 9 & 26.87 & 37.18 & 61.12 \\
\hline 10 & 28.63 & 38.47 & 69.37 \\
\hline 11 & 25.89 & 35.27 & 63.92 \\
\hline 12 & 26.70 & 37.98 & 64.94 \\
\hline 13 & 28.25 & 33.50 & 63.03 \\
\hline 14 & 29.30 & 36.54 & 65.34 \\
\hline 15 & 29.88 & 36.95 & 66.29 \\
\hline 16 & 26.95 & 35.83 & 67.36 \\
\hline 17 & 29.13 & 34.92 & 62.29 \\
\hline 18 & 27.19 & 33.24 & 70.23 \\
\hline 19 & 28.99 & 36.19 & 68.24 \\
\hline 20 & 26.03 & 35.49 & 67.25 \\
\hline 21 & 27.89 & 35.19 & 63.37 \\
\hline 22 & 28.98 & 35.20 & 69.19 \\
\hline 23 & 26.40 & 36.29 & 65.29 \\
\hline 24 & 27.09 & 36.08 & 68.30 \\
\hline 25 & 28.49 & 36.79 & 63.36 \\
\hline 26 & 26.59 & 37.59 & 61.27 \\
\hline 27 & 28.25 & 39.99 & 66.32 \\
\hline 28 & 28.20 & 38.29 & 65.25 \\
\hline 29 & 27.13 & 35.29 & 68.24 \\
\hline 30 & 27.16 & 35.79 & 67.20 \\
\hline 31 & 26.12 & 34.89 & 64.81 \\
\hline 32 & 27.39 & 38.34 & 65.29 \\
\hline 33 & 27.80 & 37.94 & 62.45 \\
\hline 34 & 28.98 & 38.46 & 65.29 \\
\hline 35 & 27.51 & 38.29 & 65.28 \\
\hline 36 & 26.00 & 39.19 & 69.29 \\
\hline 37 & 27.37 & 35.65 & 70.66 \\
\hline 38 & 28.28 & 37.98 & 68.29 \\
\hline 39 & 28.67 & 36.04 & 65.33 \\
\hline 40 & 27.39 & 34.34 & 63.39 \\
\hline 41 & 27.18 & 36.28 & 66.02 \\
\hline 42 & 26.09 & 32.40 & 64.04 \\
\hline 43 & 26.98 & 36.40 & 67.21 \\
\hline 44 & 25.79 & 35.85 & 65.32 \\
\hline
\end{tabular}

TABLE 2: Continued.

\begin{tabular}{llcc}
\hline Times & \multicolumn{2}{c}{ Quaternion representation } & $\begin{array}{c}\text { Nonquaternion } \\
\text { representation }\end{array}$ \\
& IABC & ABC & ABC \\
\hline 45 & 27.92 & 34.45 & 64.29 \\
46 & 28.79 & 35.58 & 65.28 \\
47 & 25.20 & 36.98 & 62.22 \\
48 & 26.23 & 36.55 & 68.09 \\
49 & 28.27 & 36.98 & 65.21 \\
50 & 26.68 & 34.76 & 67.90 \\
\hline Mean & 27.26 & 36.34 & 65.63 \\
\hline
\end{tabular}

images using quaternion representation, the result not only means the average running speed of IABC is faster than the $\mathrm{ABC}$, but also means that the average running speed of $A B C$ based on quaternion representation is faster than $\mathrm{ABC}$ based on the nonquaternion representation. Above all, the proposed method is faster than the other two methods. In Tables 1 and 2, $728 \times 656$ pixel region of sports center image is selected as the research subjects, and $325 \times 245$ pixel region of forest fires image is selected as the research subjects. So the data volume of sports center image is about six times as large as the one of forest fires image. But the running time of $I A B C$ based on quaternion representation in the sports center image is 4.3 times the one of IABC based on quaternion representation in the forest fires image. The running time of $A B C$ based on quaternion representation in the sports center image is 4.4 times the one of $\mathrm{ABC}$ based on quaternion representation in the forest fires image. The running time of $\mathrm{ABC}$ based on nonquaternion representation in the sports center image is 4.7 times the one of $\mathrm{ABC}$ based on nonquaternion representation in the forest fires image. This shows that one of the advantages of the proposed method with the remote sensing image data volume is a great acceleration in terms of speed. On one hand, it is because the IABC algorithm has a higher efficiency. On the other hand, it is due to the quaternion representation of color image. Vector of a color pixel is described by using only a triple array, rather than the three color separation method of the red, green, and blue. Therefore the proposed method runs faster, and results are more accurate and stable.

Tables 3-5 are the result of the three algorithms applied to the two remote sensing images in the experiment. Quantitative analysis of the effect of edge detection is shown in Tables 3-5. $A, B$, and $C$ stand, respectively, for the edge points, 4connected numbers, and 8-connected numbers. The sizes of $C / A$ and $C / B$ reflect the tightness of the edge line connection, which have a significant impact on the overall evaluation of the edges. That is to say, the values of $C / A$ and $C / B$ represent the number of error points detected and points missed in the image edge detection. Obviously, if the values of $C / A$ and $C / B$ are smaller, the edge line connection will be better and the points missed and the wrong points detected will be fewer in the image edge detection. Thus the result of edge detection is more accurate, and the effect is better. On the contrary, if the values of $C / A$ and $C / B$ are larger, the edge line connection will 
TABLE 3: ABC algorithm based on nonquaternion representation experimental data table.

\begin{tabular}{lcccc}
\hline Image & Edge points $(A)$ & 4-connected number $(B)$ & 8-connected number $(C)$ & $C / A$ \\
\hline Sports center & 126745 & 3540 & 2408 & 0.018999 \\
Forest fires & 21387 & 528 & 336 & 0.680226 \\
\hline
\end{tabular}

TABLE 4: ABC algorithm based on quaternion representation experimental data table.

\begin{tabular}{lccccc}
\hline Image & Edge points $(A)$ & 4-connected number $(B)$ & 8-connected number $(C)$ & $C / A$ & $C / B$ \\
\hline Sports center & 139681 & 3897 & 2519 & 0.018034 \\
Forest fires & 26476 & 723 & 436 & 0.646395 \\
\hline
\end{tabular}

TABLE 5: IABC algorithm based on quaternion representation experimental data table.

\begin{tabular}{lccccc}
\hline Image & Edge points $(A)$ & 4-connected number $(B)$ & 8-connected number $(C)$ & $C / A$ & $C / B$ \\
\hline Sports center & 168173 & 4467 & 2793 & 0.016608 \\
Forest fires & 320123 & 831 & 489 & 0.625252 \\
\hline
\end{tabular}

be poorer, the points missed and the wrong points detected will be the more, and the edge extraction effect will be worse. Additionally we know that the $C / B$ has a greater impact than $C / A$ on the image edge detection from the analysis of the results of edge detection. In Tables $3-5, C / A$ and $C / B$ of IABC based on quaternion representation are the smallest, which means that our method is the best in remote sensing image edge detection. In Tables 3 and $4, C / A$ in Table 3 and $C / A$ in Table 4 are almost equal in sports center image, and $C / A$ in Table 3 is slightly smaller than $C / A$ in Table 4 in forest fire image. However $C / B$ in Table 3 is significantly larger than $C / B$ in Table 4 in sports center image, and $C / B$ in Table 3 is significantly larger than $C / B$ in Table 4 in forest fires image. As $C / B$ has a greater impact than $C / A$ on remote sensing image edge detection, $\mathrm{ABC}$ algorithm based on quaternion representation has a better effect than $\mathrm{ABC}$ algorithm based on nonquaternion representation in remote sensing image edge detection; that is to say, the edge line connection is better and the points missed and the wrong points detected are fewer in the image edge detection. Further in Tables 4 and $5, C / A$ and $C / B$ in Table 5 of IABC algorithm based on quaternion representation are both smaller than $C / A$ and $C / B$ in Table 4, which means it has a better edge detection effect than $\mathrm{ABC}$ algorithm based on quaternion representation.

In short, the effect of $\mathrm{ABC}$ based on nonquaternion representation is the worst in remote sensing image edge detection. The effect of IABC algorithm based on quaternion representation is the best in remote sensing image edge detection.

\section{Conclusion}

In this paper, at the first time, we propose the quaternionbased improved $\mathrm{ABC}$ (IABC) method for color remote sensing image edge detection. By this method, when using quaternions to describe color pixel vectors, noise point becomes a singular point making it easy to separate the noise points and the general pixels. In IABC, two modified search equations are introduced to generate candidate solutions in the employed bee phase and the onlookers phase, respectively, which significantly improve the convergence speed. In addition, a more reasonable $\mathrm{ABC}$ parameter is proposed. Then the new method is used in color image. The comparison of experiment results among the new method, the quaternion-based $\mathrm{ABC}$ method, and the conventional $\mathrm{ABC}$ method shows that the new method can get a higher accuracy and be easier to suppress the noise. Above all, our method is a very competent method.

\section{Conflict of Interests}

The authors declare that there is no conflict of interests regarding the publication of this paper.

\section{Acknowledgments}

This work was supported by National Natural Science Foundation of China under Grant 61005018 and Sichuan Provincial Department of Education Youth Foundation Grant 10ZB085.

\section{References}

[1] L. Durieux, E. Lagabrielle, and A. Nelson, "A method for monitoring building construction in urban sprawl areas using object-based analysis of Spot 5 images and existing GIS data," ISPRS Journal of Photogrammetry and Remote Sensing, vol. 63, no. 4, pp. 399-408, 2008.

[2] T. Blaschke, "Object based image analysis for remote sensing," ISPRS Journal of Photogrammetry and Remote Sensing, vol. 65, no. 1, pp. 2-16, 2010.

[3] A. Tzotsos, K. Karantzalos, and D. Argialas, "Object-based image analysis through nonlinear scale-space filtering," ISPRS Journal of Photogrammetry and Remote Sensing, vol. 66, no. 1, pp. 2-16, 2011.

[4] X. Tong, Z. Hong, S. Liu et al., "Building-damage detection using pre- and post-seismic high-resolution satellite stereo 
imagery: a case study of the May 2008 Wenchuan earthquake," ISPRS Journal of Photogrammetry and Remote Sensing, vol. 68, no. 1, pp. 13-27, 2012.

[5] Z. Xie, C. Roberts, and B. Johnson, "Object-based target search using remotely sensed data: a case study in detecting invasive exotic Australian Pine in south Florida," ISPRS Journal of Photogrammetry and Remote Sensing, vol. 63, no. 6, pp. 647660, 2008.

[6] D. Karaboga, "An idea based on honey bee swarm for numerical optimization," Tech. Rep. TR06, Erciyes University, Kayseri, Turkey, 2005.

[7] M. F. Tasgetiren, Q.-K. Pan, P. N. Suganthan, and A. H. Chen, "A discrete artificial bee colony algorithm for the total flowtime minimization in permutation flow shops," Information Sciences, vol. 181, no. 16, pp. 3459-3475, 2011.

[8] V. J. Manoj and E. Elias, "Artificial bee colony algorithm for the design of multiplier-less nonuniform filter bank transmultiplexer," Information Sciences, vol. 192, pp. 193-203, 2012.

[9] J. C. Long, W. Y. Szeto, and H.-J. Huang, "A bi-objective turning restriction design problem in urban road networks," European Journal of Operational Research, vol. 237, no. 2, pp. 426-439, 2014.

[10] W. Y. Szeto and Y. Jiang, “Transit route and frequency design: bi-level modeling and hybrid artificial bee colony algorithm approach," Transportation Research B, vol. 67, pp. 235-263, 2014.

[11] A. Draan and A. Bouaziz, "Multilevel thresholding selection based on the artificial bee colony algorithm for image segmentation," Swarm and Evolutionary Computation, vol. 16, pp. 69-84, 2014.

[12] W. Szeto and Y. Jiang, "Hybrid artificial bee colony algorithm for transit network design," Transportation Research Record, no. 2284, pp. 47-56, 2012.

[13] Y. G. Sun, H. B. Huang, and J. X. Chao, "Uncertainty and processing methods to explore remote sensing image edge detection," Remote Sensing Information, no. 6, pp. 110-114, 2010.

[14] Y. B. Shao, Y. G. Zhang, Y. L. Xing, and K. Liu, "Remote sensing image edge detection based on the permutation group," Computer Simulation, vol. 27, no. 1, pp. 214-217, 2010.

[15] K. N. Plataniotis and A. N. Venetsanopoulos, Color Image Processing and Applications, Springer, Berlin, Germany, 2000.

[16] F. Pan and X. Lin, "Fast mode decision for intra prediction," in Proceedings of the JVT-G013, 7th Meeting, Pattaya,Thailand, March 2003.

[17] P. Feng and L. Xiao, "Fast mode decision for intra prediction," JVT of ISO/IEC MPEG\&ITU-T VCEG, J VI-G013, 2003.

[18] R. Lukac, B. Smolka, K. Martin, K. N. Plataniotis, and A. N. Venetsanopoulos, "Vector filtering for color imaging," IEEE Signal Processing Magazine, vol. 22, no. 1, pp. 74-86, 2005.

[19] W. R. Hamilton, Elements of Quaternions, Ginn Company, Boston, Mass, USA, 1887.

[20] F.-Y. Lang, Z. Jiliu, and Y. Bin, "Quaternion and color image edge detection," Computer Science, vol. 34, no. 11, pp. 212-216, 2007.

[21] C. E. Moxey, S. T. Sangwine, and T. A. Ell, "Hypercomplex operators and vector correlation," in Proceedings of the 11th European Signal Processing Conference (EUSIPCO '02), vol. 3, pp. 247-250, Toulouse, France, 2002.

[22] P. Denis, P. Carre, and C. Fernandez-Maloigne, "Spatial and spectral quaternionic approaches for colour images," Computer Vision and Image Understanding, vol. 107, no. 1-2, pp. 74-87, 2007.
[23] S.-C. Pei, J.-H. Chang, and J.-J. Ding, "Quaternion matrix singular value decomposition and its applications for color image processing," in Proceedings of the IEEE International Conference on Image Processing (ICIP '03), vol. 1, pp. 805-808, Barcelona, Spain, September 2003.

[24] N. Le Bihan and S. J. Sangwine, "Quaternion principal component analysis of color images," in Proceedings of the IEEE International Conference on Image Processing (ICIP '03), vol. 1, pp. 809-812, September 2003.

[25] C. E. Moxey, S. T. Sangwine, and T. A. Ell, "Hypercomplex operators and vector correlation," in Proceedings of the 11th European Signal Processing Conference (EUSIPCO '02), vol. 3, pp. 247-250, Toulouse, France, 2002.

[26] S. J. Sangwine and T. A. Ell, "Color image filters based on hypercomplex convolution," IEE Proceedings-Vision, Image and Signal Processing, vol. 147, no. 2, pp. 89-93, 2000.

[27] H. L. Jin and D. H. Li, Color Image Filtering and Color Image Processing Method Based on Quaternion, Huazhong University of Science and Technology, Hubei, China, 2008.

[28] F. Y. Lang, J. L. Zhou, B. Yan, E. B. Song, and F. Zhong, "Quaternion and color image edge detection," Computer Science, vol. 34, no. 11, pp. 212-216, 2007.

[29] J. Kennedy and R. Eberhart, "Particle swarm optimization," in Proceedings of the IEEE International Conference on Neural Networks, pp. 1942-1948, December 1995.

[30] M. Dorigo and T. Stutzle, Ant Colony Optimization, MIT Press, Cambridge, Mass, USA, 2004.

[31] K. S. Tang, K. F. Man, S. Kwong, and Q. He, "Genetic algorithms and their applications," IEEE Signal Processing Magazine, vol. 13, no. 6, pp. 22-37, 1996.

[32] D. Simon, "Biogeography-based optimization," IEEE Transactions on Evolutionary Computation, vol. 12, no. 6, pp. 702-713, 2008.

[33] F. Kang, J. Li, and Z. Ma, "Rosenbrock artificial bee colony algorithm for accurate global optimization of numerical functions," Information Sciences, vol. 181, no. 16, pp. 3508-3531, 2011.

[34] G. Zhu and S. Kwong, "Gbest-guided artificial bee colony algorithm for numerical function optimization," Applied Mathematics and Computation, vol. 217, no. 7, pp. 3166-3173, 2010.

[35] W.-F. Gao, S.-Y. Liu, and L.-L. Huang, "A novel artificial bee colony algorithm based on modified search equation and orthogonal learning," IEEE Transactions on Cybernetics, vol. 43, no. 3, pp. 1011-1024, 2013.

[36] P.-W. Tsai, J.-S. Pan, B.-Y. Liao, and S.-C. Chu, "Enhanced artificial bee colony optimization," International Journal of Innovative Computing, Information and Control, vol. 5, pp. 1-12, 2009.

[37] W.-F. Gao and S.-Y. Liu, "A modified artificial bee colony algorithm," Computers and Operations Research, vol. 39, no. 3, pp. 687-697, 2012.

[38] J. Tian, W. Yu, and S. Xie, "An ant colony optimization algorithm for image edge detection," Proceedings of the IEEE World Congress on Computational Intelligence, 2008.

[39] R. Lin and N. Shu, "A new evaluation method based on edge connectivity omponents," Remote Sensing of Land and Resources, vol. 3, pp. 37-40, 2003. 


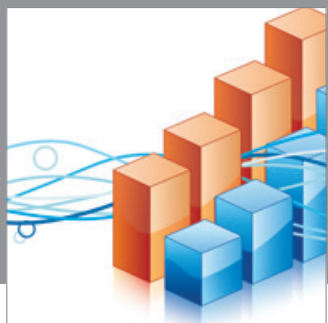

Advances in

Operations Research

mansans

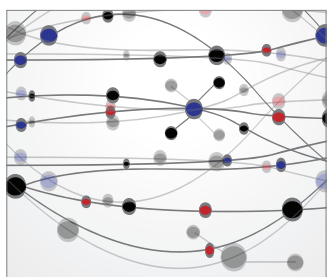

The Scientific World Journal
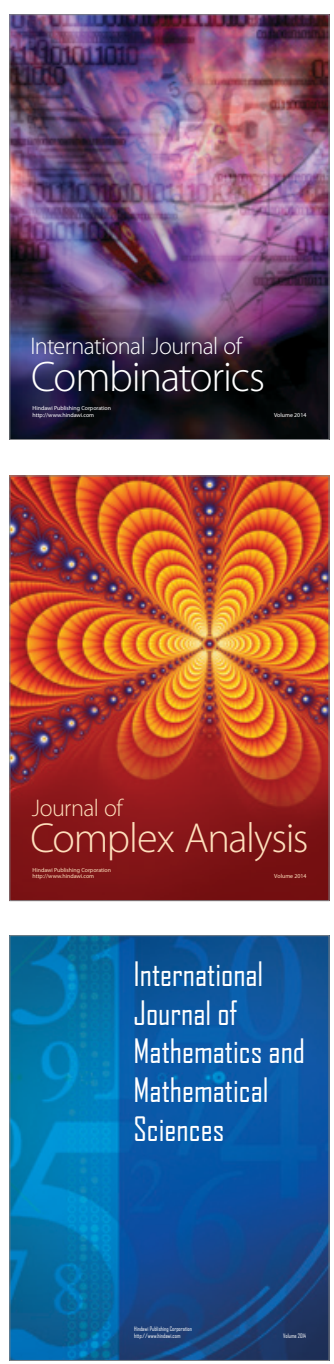
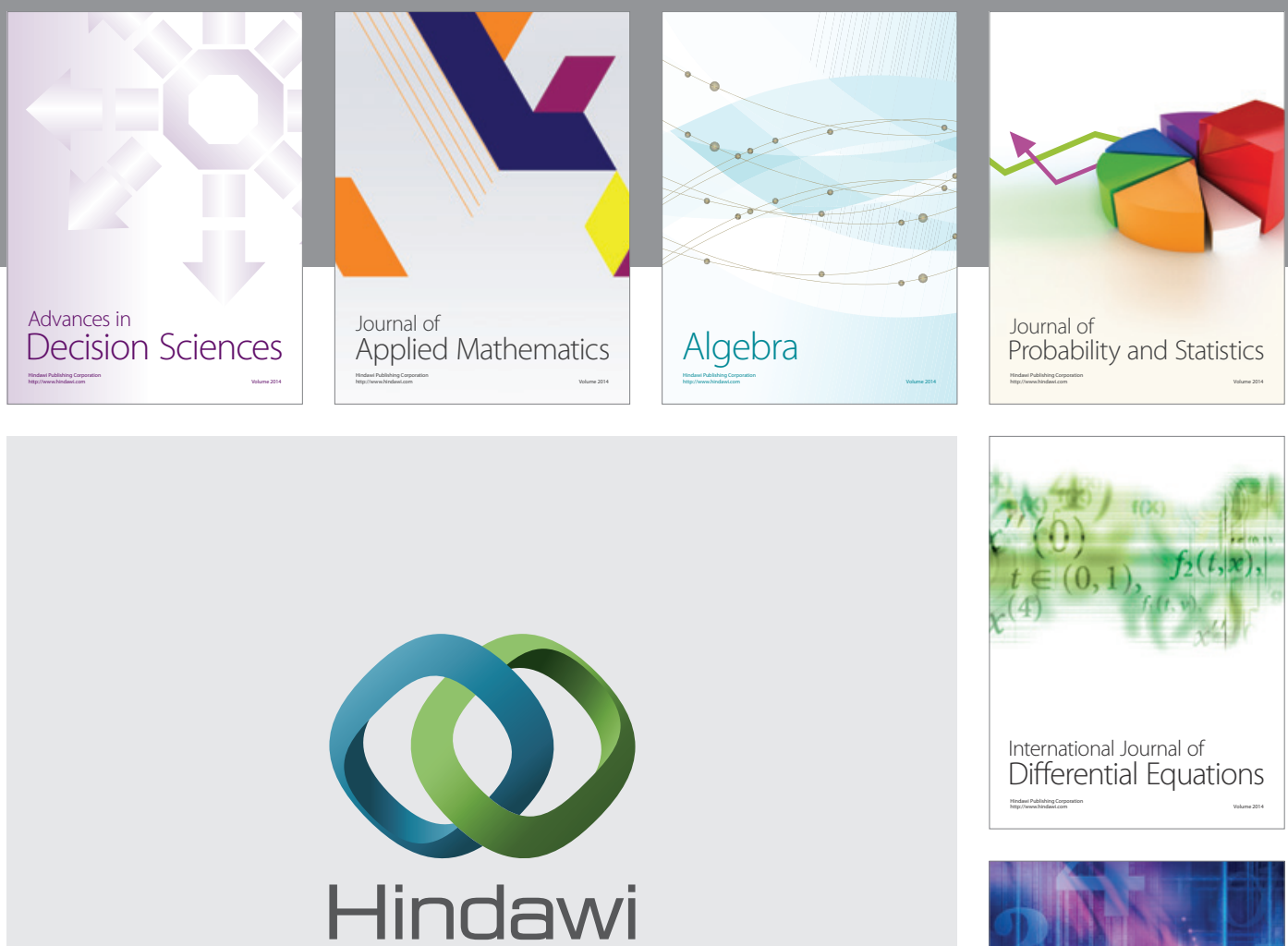

Submit your manuscripts at http://www.hindawi.com
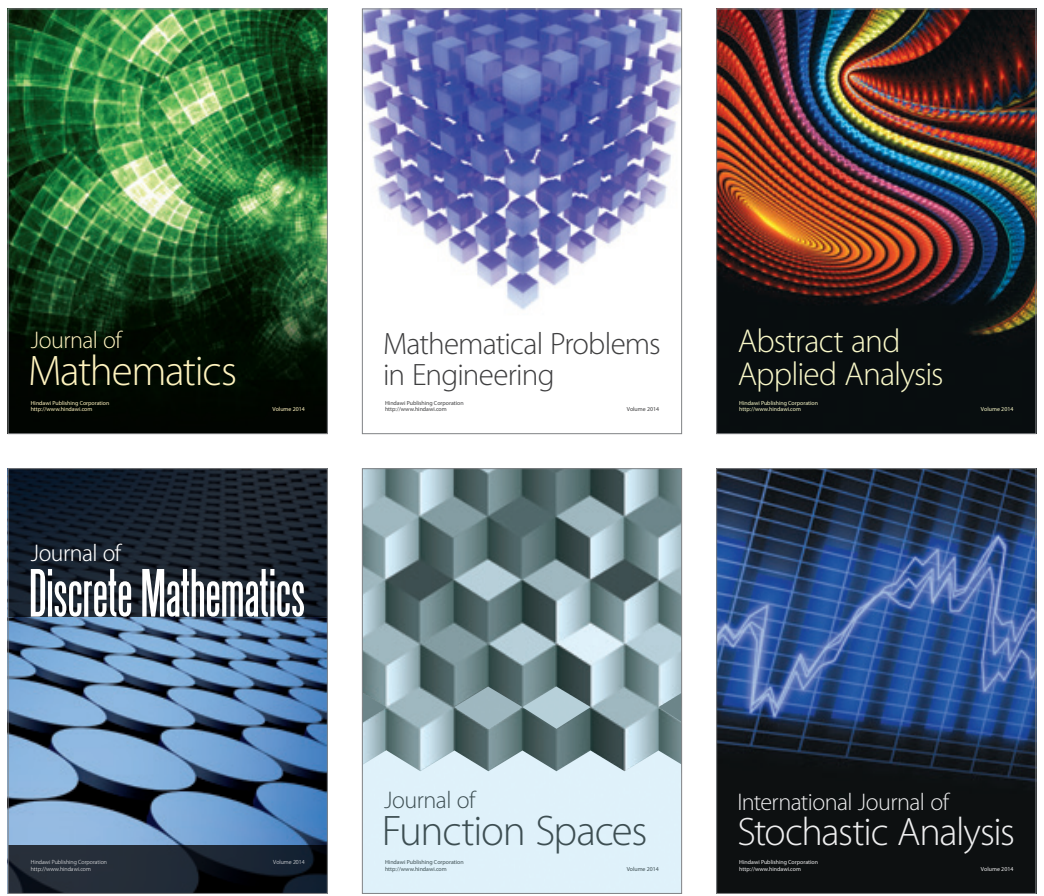

Journal of

Function Spaces

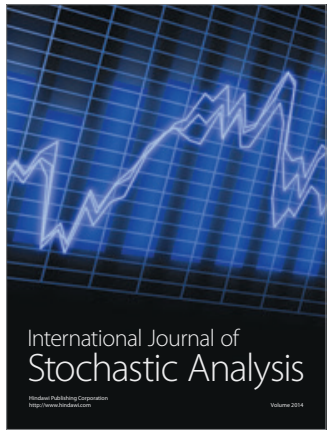

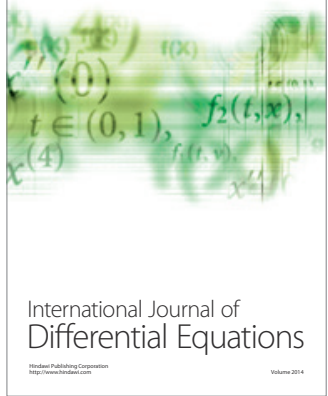
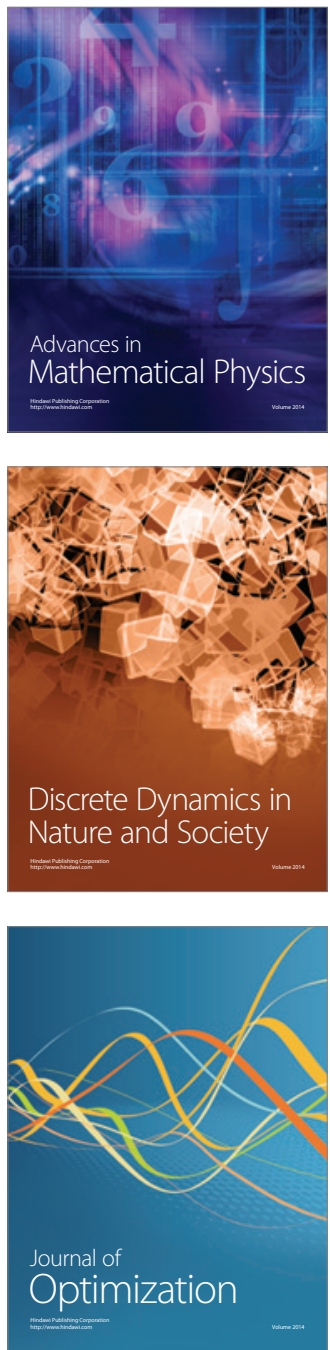